\title{
Epidemiology of Extended Spectrum Beta Lactamase Producing Gram Negative Rods
}

\author{
Ayesha Rafique, Farhan Rasheed, Muhammad Saeed, Sajjad Hassan, Syeda Fatima Nadeem,
} Ambereen Anwar Imran

\begin{abstract}
OBJECTIVE: To estimate the distribution of ESBL producing capacity among different Gram negative isolates along with specimen and department wise prevalence in a tertiary care hospital.

METHODOLGY: This cross-sectional study was conducted at the Microbiology section of Pathology Department, Allama Iqbal Medical College, Lahore, Pakistan, from August 2016 to January 2017. Total of 437 clinical samples were collected from different wards of Jinnah Hospital, Lahore. Sample was cultured on Blood agar, MacConkey's agar, chocolate agar, CLED agar. After the identification every Gram negative isolate was further processed for the antibiotic susceptibility testing following Modified Kirby Bauer disc diffusion method. ESBL detection was performed by the combination drugs disc method using ceftriaxone + clavulanic acid $(30 / 10 \mu \mathrm{g})$ in case of Enterobacteriacae, ceftazidime + clavulanic acid $(30 / 10 \mu \mathrm{g})$ in case of Pseudomonas species.

RESULTS: Among total 437 patients male and female were $61 \%$ and $39 \%$ respectively, overall $21.5 \%$ (94/437) were ESBI producers. Distribution of ESBL producers was as followed Acinetobacter spp $22.3 \%$ (31/139), Escherichia coli $37.5 \%$ (27/72), Klebsiella spp 31.9\% (15/47), Pseudomonas aeruginosa $8.4 \%$ $(13 / 154)$ and Proteus spp 32.0\% (8/25). Department wise ESBL positivity was as followed Surgical units $25.6 \%$ (32/125) Medical unit 31.9\% (30/94), Burn centre 10\% (8/80), ICU 20\% (8/40) Surgical Allied $11.7 \%$ (4/34), Medical Allied 6.2\% (2/32), Neurology 36.3\% (8/22), Gynaecology $28.5 \%$ (2/7) and Dermatology $33.3 \%(1 / 3)$.

CONCLUSION: High percentage of ESBL positivity seen in Escherichia coli and Proteus spp, among departments neurology and medical unit is holding maximum positivity. High frequency of ESBL producing Gram negative isolates strongly recommend its detection and management accordingly. It can lead to treatment failures in Gram negative rods associated infections.
\end{abstract}

KEYWORDS: ESBL, Gram Negative Rods, Escherichia coli.

This article may be cited as: Rafique A, Rasheed F, Saeed M, Hassan S, Nadeem SF, Imran AA. Epidemiology of Extended Spectrum Beta Lactamase Producing Gram Negative Rods. J Liaquat Uni Med Health Sci. 2019;18(02):164-8. doi: 10.22442/jlumhs.191820621

\section{INTRODUCTION}

Inspite of several solid steps taken to reduce the emergence of drug resistance, still it is prime threat, our hospitals are full of infected people with no appropriate treatment due to drug resistant isolates, endangering the efficacy of antibiotics ${ }^{1,2}$. Clinicians have nothing for them but wait, pray and watch them die. Extensive and inappropriate use of $\beta$-lactam drugs in our health care settings has twisted foremost resistance problem leading to increase in health care cost, morbidity and mortality in all over the world. Production of $\beta$-lactamases is the most common cause of drug resistance against $\beta$-lactam group of antibiotics. There are more than 1300 beta lactamases in the arsenal of these enzymes and extended spectrum $\beta$-lactamases (ESBL) is one of them $^{3}$.

ESBL has generally been defined as transmissible beta-lactamases resistant to $3^{\text {rd }}$ generation cephalosporin, monobactam that can be inhibited by clavulanic acid, tazobactam or sulbactam which are encoded by gene that can be exchanged between bacteria ${ }^{4}$. ESBL has generally been a group of plasmid mediated, diverse, complex and rapidly evolving emergency that is posing a major therapeutic challenges in the hospital acquired and tertiary care setups. ESBL majorly comes in the class $B$ of AMBLER'S molecular classification ${ }^{5}$. ESBL lies in 2be, 2ber, 2de, 2e, according to Bush-Jacoby functional classification ${ }^{6}$.

Detection of ESBL is tough job in developing countries $^{7}$. According to few studies almost $37 \%$ of ESBL producers were misreported $^{8}$. Moreover according to Tenover FC $1999^{9}$ out of 38 laboratories only 07 were correctly identified and reported ESBL. These scenarios suggest that perfection in the skill of clinical laboratories to detect ESBL is required. 
Therefore detection of ESBL is routinely involved in routine practice, which may lead to result in misreporting and henceforward treatment failures. Furthermore in low ESBL endemic areas it may not be cost effective to test for ESBL on a routine basis.

ESBL detection usually done via phenotypic and genotypic methods. Phenotypic methods include MIC by E-Strip, Agar dilution and by broth dilution ${ }^{10}$. The most common and sensitive method of genotypic detection of ESBL is PCR (Polymerase Chain Reaction). Many types of PCR are involved in the detection of ESBL i.e Duplex, Multiplex, Real-time, Pyro-sequencing, Reverse-line hybridization ${ }^{11}$.

Detection of ESBL is a paramount as it provides clinicians with supportive information. Although ESBL producers may showed susceptibility to extended spectrum drugs i.e Cephalosporin or Aztreonam but they are intrinsingly resistant. Moreover the patients inhabited or infested with ESBL producer should be isolated under contact precautions to avoid spread of nosocomial infections. These benefits summon the detection of ESBL-producing organisms in clinical laboratories. Clinically the treatment option for ESBL producing isolates is limited to Carbapenems among beta lactam drugs. Spectrum of ESBL producer differs in accordance with geographic location ${ }^{7}$. Therefore present study was conducted to determine the spectrum of ESBL producers Gram negative rods among clinical specimens received from different departments of a tertiary care hospital.

\section{METHODOLOGY}

This cross-sectional study was conducted at Microbiology section of pathology department at Allama Iqbal Medical College, Lahore, Pakistan, from August 2016 to January 2017.Non probability consecutive sampling technique was used and a total of 439 clinical samples were collected from different wards of Jinnah hospital, Lahore.

All clinical samples both from male and female patients from indoor/admitted patients, Gram negative rods resistant to $3^{\text {rd }}$ generation cephalosporin were included while duplicate samples from same patient during same episode of illness, all Gram negative rods susceptible to $3^{\text {rd }}$ generation cephalosporin were excluded. Every sample was processed according to standard protocol and cultured on Blood agar, MacConkey's agar, Chocolate agar, CLED agar. Organisms were identified on the basis of colonial morphology (Lactose fermenter, non-lactose fermenter, late lactose fermenter, size, color of colony) Gram staining reaction and biochemical tests i.e. oxidase test, catalase test, Triple sugar iron, Citrate utilization test, Urease test, Indole test, semisolid motility agar.
ESBL: Detection by combination drugs disc method After the confirmation, every Gram negative isolate was further processed for the antibiotic susceptibility testing following Modified Kirby Bauer disc diffusion method, ESBL detection was performed by the combination drugs disc method using ceftriaxone + clavulanic acid $(30 / 10 \mu \mathrm{g})$ in case of Enterobacteriaseae and ceftazidime + clavulanic acid $(30 / 10 \mu \mathrm{g})$ in case of Pseudomonas species. Results were interpreted as followed, zone size of ceftriaxone + clavulanic acid disc is $5 \mathrm{~mm}$ bigger than the zone size of ceftriaxone $(30 \mu \mathrm{g})$ disc was tagged as ESBL producer while the zone size of ceftriaxone + clavulanic acid disc is not $5 \mathrm{~mm}$ bigger than the zone size of ceftriaxone $(30 \mu \mathrm{g})$ disc were considered as non ESBL producer. Same interpretation protocol was adopted for ceftazidime + calvulanic discs.

Data was entered in SPSS version 21.0 and descriptive analysis was done.

\section{RESULTS}

Among total 439 patients male and female were $61 \%$ and $39 \%$ respectively. Distribution of these $3^{\text {rd }}$ Generation cephalosporin resistant isolates was as followed Acinetobacter spp 31.6\%, Escherichia coli $16.4 \%$, Klebsiella spp 10.7\%, Pseudomonas aeruginosa $35.0 \%$ and Proteus spp $5.6 \%$. While distribution of ESBL producers was as followed Acinetobacter spp 22.3\% (31/139), Escherichia coli $37.5 \%$ (27/72), Klebsiella spp 31.9\% (15/47), Pseudomonas aeruginosa 8.4\% (13/154) and Proteus spp $32.0 \%$ (8/25) Figure I.

Figure II depicted department wise distribution of total samples and ESBL producers, maximum number of samples were received from surgical units $28.6 \%$ (12/437), followed Medical unit 21.5\% (94/437), Burn centre 18.3\% (80/437), ICU 9.1\% (40/437) Surgical Allied 7.7\% (34/437), Medical Allied 7.3\% (32/437), Neurology 5.0\% (22/437), Gynecology 1.6\% (7/437) and Dermatology $0.6 \%$ (3/437). ESBL positivity was followed surgical units 25.6 (32/125), followed Medical unit $31.9 \%$ (30/94), Burn centre 10\% (8/80), ICU 20\% (8/40) Surgical Allied 11.7\% (4/34), Medical Allied $6.2 \%(2 / 32)$, Neurology $36.3 \%(8 / 22)$, Gynecology $28.5 \%(2 / 7)$ and Dermatology 33.3\% (1/3) Figure II.

\section{FIGURE I: ORGANISMS BASED FREQUENCY DISTRIBUTION OF ESBL PRODUCERS $(n=437)$}

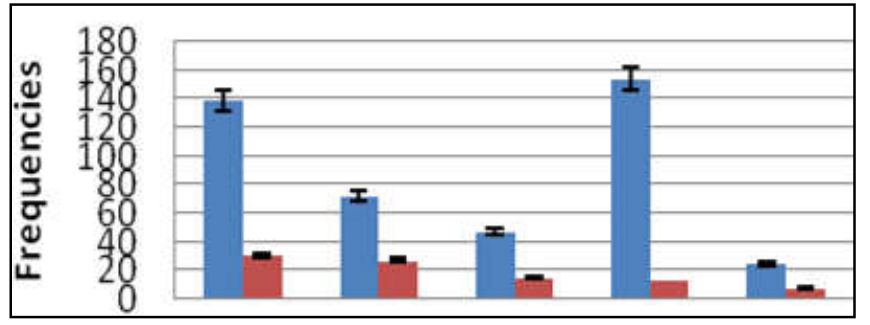




\section{FIGURE II: DEPARTMENT WISE FREQUENCY DISTRIBUTIONS OF ESBL PRODUCERS ( $n=94)$}

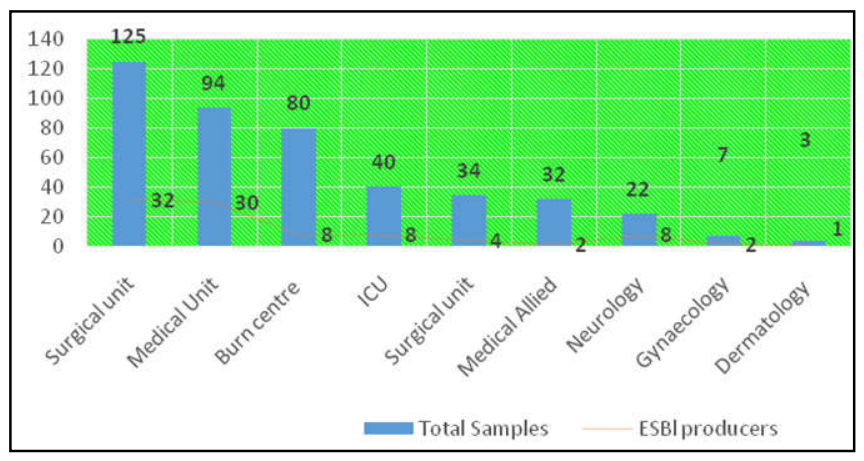

DISCUSSION

Since 1970's when the first description was done, drug resistance surveillance has a key role among all policies to control the problem of drug resistance ${ }^{12}$. Globally ESBL have been isolated and form a major contributor of antimicrobial resistance in Gram negative isolates ${ }^{13}$. Now a days ESBL producers are prime threat as nosocomial infections for hospitalized patients throughout around the globe ${ }^{14}$. Frequency and distribution of ESBL producers among different clinical samples vary greatly around the globe. Present study reported $21.4 \% \quad(n=94)$ ESBL producers, Acinetobacter spp 22.3\%, Escherichia coli $37.5 \%$, Klebsiella spp $31.9 \%$, Pseudomonas aeruginosa $8.4 \%$ and Proteus spp $32.0 \%$ Figure I. Similarly Ali AM $2004^{15}$ from Army Medical College, Rawalpindi reported very high rate of ESBL producers i.e; $45 \%(n=366)$ Escherichia coli with $45 \%$ was the most frequent organism isolated followed by Klebsiella pneumoniae with $21 \%$, Pseudomonas aeruginosa with 19.2\%, Enterobacter cloacae 4.6\% and Acinetobacter baumannii 4.4\% Multiple studies reported similar results i.e Ahmad N 2016 ${ }^{16}$ reported out of 209 Enterobacteriaceae isolates, $32 \%$ were ESBL producers, Enterobacter species 47.8\% Klebsiella species $40 \%$ and E. coli $29.5 \%$. Kausar A et al ${ }^{17}$ reported out of 199 Gram negative isolates $61 \%$ were ESBL producers, E. coli 56\% Pseudomonas aeruginosa $18 \%$, Klebsiella species $17 \%$ and Proteus species $8 \%$. Ghazal L $2015^{18}$ reported frequency of ESBL producing organisms 66\%, among these GNRs, Klebsiella pneumoniae 52\%, Escherichia coli $38 \%$, Enterobacter cloacae 4.6\%, Citrobacter freundii 3.6\% and Proteus mirabilis $0.9 \%$. Hafeez R $2009^{19}$ reported $35 \%$ ESBL producers, with the highest frequency of Escherecia coli 44\%, followed by Klebsiella pneumoniae $38 \%$, Proteus mirabilis $31 \%$ and Acinetobacter baumannii $7 \%$. Jabeen K $2005^{7}$ reported $40 \%$ ESBL producers , $71 \%$ E. coli, $15 \% \mathrm{~K}$. pneumoniae and $9 \%$ Enterobacter species. Sasirekha B $2010^{20}$ reported $65.8 \%$ ESBL producers with maximal incidence in $E$. coli $73.8 \%$ followed by Klebsiella pneumoniae 51.1\%. Rahman MM $2004^{21}$ used double disk test to detect ESBL production, and reported Escherichia coli and Klebsiella pneumoniae $43.2 \%$ and $39.5 \%$ respectively. Jain et al from India reported distribution of ESBL producers as followed Klebsiella spp $87.2 \%$, Enterobacter spp $72.5 \%$, E. coli $65.3 \%$ and Acinetobacter spp $33.3 \%$. and in none of the isolates of Citrobacter or Pseudomonas $\mathrm{spp}^{22}$.

Excessive and imprudent use of antibiotics, lack of the knowledge for the development of new formula drugs by the pharmaceutical industries contributes in the emergence of bacterial resistance ${ }^{23}$. Although drug resistance is naturally observed in bacterial isolates of remote places irrespective of excessive human intervention of antibiotics. However antibiotic biosynthetic genes and resistance-conferring genes are also involved. Other mechanisms of drug resistance include, enzyme production causes inactivation of drug, modification of target sites, mutation in porin proteins and efflux pump ${ }^{24}$.

Bacteria produces enzymes ß-lactamases inactivates beta lactam drugs by cleaving the ß-lactam ring of the drug and inactivate antimicrobial drug. Modified target sites are developed against which the drug has no effect. A mutant protein in the $30 \mathrm{~S}$ ribosomal subunit can result in resistance to certain, and a methylated 23S rRNA can result in resistance to many antibiotics $^{25}$. Bacteria decreases their cell membrane permeability so that effective intracellular concentration of the drug should not achieved such changes in porin proteins of cell membrane can reduce the amount of certain antibiotics to enter bacterium causes antibiotic resistance. Bacteria actively export drugs using a multidrug resistance pump or efflux pump that import protons and in an exchange-type reaction, export a variety of foreign molecules including certain antibiotics results in no antibiotic effect on the active site.

Genetic mechanisms of drug resistance include chromosome-mediated resistance, plasmid-mediated resistance, and transposon-mediated resistance ${ }^{4}$. CLSI recommended methods of screening of ESBL production are not much sensitive and can detect false positive ESBL producers. Double disc synergy method which CLSI recommends for epidemiological studies should be used in routine for detection of ESBL. Because treatment options are limited for ESBL producing Gram negative rods and are difficult to treat.

\section{CONCLUSION}

High percentage of ESBL positivity seen Escherichia coli and Proteus spp, among departments of 
neurology and medical unit is holding maximum positivity. High frequency of ESBL producing Gram negative isolates strongly recommend its detection and management accordingly. It can lead to treatment failures in Gram negative rods associated infections.

Ethical Approval: AIMC/JHL Ref. No. 34thA.12/ERB Dated. 27-10-2016.

Conflict of interest: There was no any conflict of interest.

Funding: There was no any funding agency.

\section{REFERENCES}

1. Golkar Z, Bagasra O, Pace DG. Bacteriophage therapy: a potential solution for the antibiotic resistance crisis. J Infect Dev Ctries. 2014; 8(2): 129-36. doi: 10.3855/jidc.3573.

2. Ventola CL. The antibiotic resistance crisis: part 2: management strategies and new agents. Pharm Therapeut. 2015; 40(5): 344-52.

3. Fankhauser C, Schrenzel J, Prendki V, Ris F, Schiffer E, Gastmeier P, et al. Prevalence of extended-spectrum betalactamase producingEnterobacteriaceae (ESBL-E) carriage on admission at Geneva University Hospitals (HUG). Antimicrob Resist Infect Control. 2015; 4(S1): P120. doi: 10.1186/2047-2994-4-S1-P120

4. Shaikh S, Fatima J, Shakil S, Rizvi SM, Kamal MA. Antibiotic resistance and extended spectrum beta-lactamases: Types, epidemiology and treatment. Saudi J Biol Sci. 2015; 22(1): 90-101. doi: 10.1016/j.sjbs.2014.08.002.

5. Adwan G, Jaber AA. Frequency and Molecular Characterization of $\beta$-lactamases Producing Escherichia coli Isolated from North of Palestine. Br Microbiol Res J. 2016; 11(5): 1-13.

6. King AM. Discovery and Characterization of Novel Beta-Lactamase Inhibitors.[Phd Thesis]. Ontario, Canada: McMaster University. 2016.

7. Jabeen K, Zafar A, Hasan R. Frequency and sensitivity pattern of Extended Spectrum beta Lactamase producing isolates in a tertiary care hospital laboratory of Pakistan. J Pak Med Assoc. 2005; 55(10): 436-9.

8. Livermore D, Yuan M. Antibiotic resistance and production of extended-spectrum $\beta$-Mactamases amongst Klebsiella spp. from intensive care units in Europe. J Antimicrob Chemother. 1996; 38(3): 409-24. 10.1093/jac/38.3.409.

9. Tenover FC, Mohammed MJ, Gorton TS, Dembek ZF. Detection and reporting of organisms producing extended-spectrum $\beta$-lactamases: survey of laboratories in Connecticut. J Clin Microbiol. 1999; 37(12): 4065-70.

10. Poulou A, Grivakou E, Vrioni G, Koumaki V,
Pittaras T, Pournaras $S$, et al. Modified CLSI extended-spectrum $\beta$-lactamase (ESBL) confirmatory test for phenotypic detection of ESBLs among Enterobacteriaceae producing various $\beta$-lactamases. J Clin Microbiol. 2014; 52 (5): 1483-9. doi:10.1128/JCM.03361-13.

11. Güzel M, Genç $Y$, Aksoy A, Moncheva $P$, Hristova $P$. Investigation of three different methods for detection of ESBL production and antibiotic resistance percentage of ESBL producing Gram negative bacteria. Turk Bull Hygiene Exp Biol. 2015; 72(2):131-8. doi:10.5505/TurkHijyen. 2015.33239

12. Doll $M$, Hewlett $A L$, Bearman $G$. Infection Prevention in the Hospital from Past to Present: Evolving Roles and Shifting Priorities. Curr Infect Dis Rep. 2016; 18: 16-19. doi:10.1007/s11908016-0523-z

13. Dortet L, Poirel L, Nordmann P. Worldwide dissemination of the NDM-type carbapenemases in Gram-negative bacteria. BioMed Res Int. 2014; 2014: 249856. doi: 10.1155/2014/249856

14. Szél B, Reiger Z, Urbán E, Lázár A, Mader K, Damjanova I, et al. Successful elimination of extended-spectrum beta-lactamase (ESBL)producing nosocomial bacteria at a neonatal intensive care unit. World J Pediatr. 2017; 13(3): 210-216. doi: 10.1007/s12519-016-0069-z.

15. Ali AM, Rafi S, Qureshi AH. Frequency of extended spectrum beta lactamase producing gram negative bacilli among clinical isolates at clinical laboratories of Army Medical College, Rawalpindi. J Ayub Med Coll Abbottabad. 2004; 16(1): 35-7

16. Ahmed N, Kamal M, Kamran A, Zaidi AH. Frequency of Extended Spectrum Beta Lactamases in Enterobacteriaceae in Urinary Isolates related to Age and Gender. Med Channel. 2016; 22: 16-20.

17. Kausar A, Akram M, Shoaib M, Mehmood RT, Abbasi MN, Adnan $M$, et al. Isolation and identification of UTI causing agents and frequency of ESBL (Extended Spectrum Beta Lactamase) in Pakistan. Am J Phytomed Clin Therap. 2014; 2 (8):963-75.

18. Ghazal L, Qureshi AH, Tehseen T. Frequency of Extended Spectrum Beta Lactamases Producing Gram Negative Bacilli among Clinical Isolates in Tertiary Care Hospital at Wah. Infect Diseases J Pak. 2015; 24(3): 850-54.

19. Hafeez R, Aslam M, Mir F, Tahir M, Javaid I, Ajmal AN. Frequency of extended spectrum beta lactamase producing gram negative bacilli among clinical isolates. Biomedica. 2009; 25(2): 112-115.

20. Sasirekha B, Manasa R, Ramya P, Sneha R. 
Frequency and antimicrobial sensitivity pattern of extended spectrum $\beta$-lactamases producing $E$. coli and Klebsiella pneumoniae isolated in a tertiary care hospital. Al Ameen J Med Sci. 2010; 3(4): 265-71.

21. Rahman MM, Haq JA, Hossain MA, Sultana R, Islam $F$, Islam AH. Prevalence of extended-spectrum $\beta$-lactamase-producing Escherichia coli and Klebsiella pneumoniae in an urban hospital in Dhaka, Bangladesh. Int J Antimicrob Agents. 2004; 24(5): 508-10.

22. Jain A, Roy I, Gupta MK, Kumar M, Agarwal SK. Prevalence of extended-spectrum $\beta$-lactamaseproducing Gram-negative bacteria in septicaemic neonates in a tertiary care hospital. J Med
Microbiol. 2003; 52(Pt 5): 421-5.

23. Haque M. Antimicrobial Use, Prescribing, and Resistance in Selected Ten Selected Developing Countries: A Brief Overview. Asian J Pharm Clin Res. 2017;10(8):37-45. doi: 10.22159/ajpcr.2017. v10i8.19468

24. Mo $Q$, Lou $T$, Wang L, Zhong $H$, Dong $Y$, Ma $D$, et Al. Molecular Mechanism of Drug Resistance of Non-Fermentative Gram-Negative Bacteria in Hospitals. Int J Clin Exp Pathol. 2016; 9(3): 358692.

25. Blair JM, Webber MA, Baylay AJ, Ogbolu DO, Piddock LJ. Molecular mechanisms of antibiotic resistance. Nat Rev Microbiol. 2015; 13(1): 42-51. doi: $10.1038 /$ nrmicro3380.

\section{AUTHOR AFFILIATION:}

\section{Ayesha Rafique}

Medical Lab Technologist

Department of Pathology

Shaukat Khanum Memorial Cancer Hospital and Research Center Lahore, Punjab-Pakistan.

\section{Dr. Farhan Rasheed}

Assistant Professor

Department of Pathology (Microbiology Section)

Allama Iqbal Medical College \& Jinnah Hospital (AIMC\&JHL)

Lahore, Punjab-Pakistan.

\section{Muhammad Saeed (Corresponding Author)}

Medical Lab Technologist

Department of Pathology DHQ Hospital

Mandi Bahauddin, Punjab-Pakistan.

Email: mian.muhsaeed@gmail.com

\section{Sajjad Hassan}

Doctor of Medical Laboratory Sciences, University Institute of Medical Laboratory Sciences

The University of Lahore, Punjab-Pakistan.

\section{Syeda Fatima Nadeem}

M.Phil Scholar

Department of Microbiology

Government College University

Lahore, Punjab-Pakistan.

\section{Prof. Ambereen Anwar Imran}

Department of Pathology

Allama Iqbal Medical College Lahore, Punjab-Pakistan. 